\title{
Remitting seronegative symmetrical synovitis with pitting edema (RS3PE): A case report and brief review
}

Remitting seronegative symmetrical synovitis with pitting edema (RS3PE) is a rare form of inflammatory arthritis, most often affecting elderly individuals and characterized by polyarthritis most commonly affecting the hands and feet in a symmetric fashion, associated with pitting edema on the dorsum of the hands (and occasionally the feet). Symptoms often spontaneously remit and are usually very responsive to low dose glucocorticoids. RS3PE has been considered as forme fruste of polymyalgia rheumatica. It can also occur as a paraneoplastic syndrome and can sometimes be a harbinger of malignancy. This article describes a case of RS3PE, followed by a review of literature about this very intriguing rheumatologic disease.

Keywords: Remitting seronegative symmetrical synovitis with pitting edema (RS3PE) • polyarthritis

\section{Introduction}

Remitting seronegative symmetrical synovitis with pitting edema (RS3PE) is a rare form of inflammatory arthritis, typically affecting elderly men with a clinical picture of symmetric polyarthritis and pitting edema on the dorsum of hands, and sometimes the feet. The pathophysiology of RS3PE is not clearly understood. Several reports have considered this to be a form of polymyalgia rheumatica and even seronegative rheumatoid arthritis, but several differences in the clinical presentation and pathophysiologic mechanisms indicate that it may be a separate entity. Once a diagnosis of RS3PE is made, it should prompt consideration of evaluation for underlying malignancy, as it can often present in the form of a paraneoplastic syndrome. This article presents a case of RS3PE followed by a brief review of literature.

\section{Case presentation}

An 85-year-old gentleman with a past medical history significant for hypertension, gout, hyperlipidemia, right femur fracture treated with internal fixation and bladder cancer treated with resection and intravesicular Bacillus CalmetteGuerin, presented with a 3-month history of sudden onset and slowly worsening pain and swelling in his hands, along with milder pain and stiffness in his shoulders, hips and thighs. Additionally, he noted generalized myalgia and overall stiffness in the morning. Prior to the onset of these symptoms, he led an active lifestyle including frequent kayaking and bicycling, and regular exercise at the gym. At presentation, he reported difficulty with daily tasks like gripping objects and opening doorknobs.

On examination, he had swelling restricted to the dorsum of both hands with pitting edema, without any signs of synovitis. He had mild restriction on internal rotation in his right shoulder associated with pain, but otherwise had no significant difficulty with shoulder or hip movements. The remainder of his exam was fairly unremarkable.

Laboratory testing revealed negative Rheumatoid Factor (RF), anti-Cyclic Citrullinated Peptide (anti-CCP) and Anti-Nuclear Antibody (ANA), normal Thyroid-Stimulating Hormone (TSH), mild anemia with hemoglobin of $11.8 \mathrm{~g} / \mathrm{dL}$, elevated erythrocyte sedimentation rate (ESR) $69 \mathrm{~mm} / \mathrm{hr}$ and elevated ZC-Reactive Protein (CRP) $5.5 \mathrm{mg} / \mathrm{dL}$. Plain radiographs of both hands showed soft tissue swelling, changes consistent with osteoarthritis and no erosions. The overall presentation raised concern of RS3PE. Treatment with prednisone $15 \mathrm{mg}$ daily was instituted.

At a follow up visit one week later, dramatic improvement of all his symptoms was noted. The swelling on the dorsum of his hands had completely resolved. The ESR and CRP returned to normal. At a 4 week follow up visit,
Rajandeep Paik ${ }^{* 1,2}$

'Lecturer in Medicine, Harvard Medical School, Massachusetts, USA ${ }^{2}$ Cambridge Health Alliance, USA

*Author for correspondence: dr.rajandeep.singh@hotmail.com 
he remained asymptomatic, the anemia had resolved and ESR and CRP remained normal. At this point, tapering of prednisone dose was started. Comprehensive search for underlying malignancy was negative, including CA 199, carcinoembryonic antigen, prostate specific antigen, serum and urine protein electrophoresis and computed tomography scans of his chest, abdomen and pelvis. His previously treated bladder cancer was followed by his urologist and cystoscopy was normal.

\section{Discussion}

Remitting seronegative symmetrical synovitis with pitting edema is a rare autoimmune condition. The first description was in 1985 in a series of 10 patients by McCarthy et al. when it was believed to be a subset of seronegative rheumatoid arthritis [1].

Over time, several studies have demonstrated a male preponderance and age of onset commonly 70 years or older [2]. Bilateral and symmetric hand involvement is the most common presentation, although occasionally symptoms can be asymmetric or unilateral, and other joints including feet, ankles and shoulders can be involved. Constitutional symptoms including fever, malaise, fatigue and weight loss can be seen. Although the name includes the term synovitis, the most characteristic finding is tenosynovitis of the extensor tendons of the hands, RS3PE has been described in association with other rheumatologic disease including sarcoidosis [3], gout [4], Sjogren syndrome [5] and polymyalgia rheumatica [6,7].

RS3PE has also been observed in the form of a paraneoplastic syndrome in association with solid organ malignancies, and rarely, hematological malignancies [8]. It may occasionally be the harbinger of an underlying malignancy.

It has been postulated that RS3PE may be a subset of Rheumatoid Arthritis (RA), but is now felt to be an entity distinct from RA, based on lack of erosion, seronegativity, excellent prognosis without the use of Disease Modifying Anti-Rheumatic Drugs (DMARDS) and absence of the Human Leukocyte Antigen (HLA) DRB1 genotype [9].

Similarly, RS3PE has been considered by some authors to be a forme fruste of polymyalgia rheumatica (PMR), based on several similarities including older age of onset, elevated acute phase reactants and excellent response to glucocorticoids [10,11]. However, there are some distinctive features including male preponderance, involvement predominantly of distal extremities, shorter duration of disease and significantly lower rates of recurrence. Based on these, RS3PE indeed appears to be an entity separate from PMR.

Acute phase reactants including ESR and CRP are elevated in the majority of patients. RF and anti-CCP are negative by definition. Some cases have noted positive ANA in low titers. Anemia and thrombocytosis can be observed, as in any inflammatory condition. Prior studies looked at elevation of serum Vascular Endothelial Growth Factor (VGEF) and this may be associated with the pathogenesis of RS3PE and may have a future role in diagnosis and management [12].

However, a recent multicenter study found that VGEF level increase in general in rheumatic diseases and this was not associated with specific rheumatic diseases [13]. Plain radiographs do not show erosive disease. Modalities like ultrasound and Magnetic Resonance Imaging (MRI) often shows tenosynovitis in extensor tendons [14].

The authors of a systematic review and metaanalysis in 2016 proposed the following criteria for diagnosis:

- Abrupt onset

- Marked pitting edema of hands (and/or feet)

- Age of onset $\geq 60$ years

- Good response to short course of medium dose steroids $(10-20 \mathrm{mg})$

- Absence of radiographic joint erosions [2].

\section{Conclusion}

Most patients respond very well to low to moderate doses of glucocorticoids. In terms of tapering, an approach similar to that in PMR can be followed, with beginning of taper in 4-6 weeks after control of symptoms and normalization of acute phase reactants. The presence of malignancy at the time of diagnosis and solid organ tumors were associated with a higher incidence of recurrence after treatment. Symptoms of paraneoplastic RS3PE have been shown to disappear after successful treatment of underlying malignancy.

The decision to perform extensive screening for underlying malignancy should be individualized, based on presence/absence of signs or symptoms of malignancy, cost-effectiveness, unusual features like lack of response to low-medium dose glucocorticoids and history of prior malignancy. 


\section{References}

1. McCarty DJ, O'Duffy JD, Pearson L et al. Remitting seronegative symmetrical synovitis with pitting edema. RS3PE syndrome. JAMA. 254(19), 2763-2767 (1985).

2. Karmacharya P, Donato AA, Aryal MR et al. RS3PE revisited: a systematic review and metaanalysis of 331 cases. Clin. Exp. Rheumatol. 34(3), 404-415 (2016).

3. Matsuda M, Sakurai K, Fushimi T et al. Sarcoidosis with high serum levels of vascular endothelial growth factor (VEGF), showing RS3PE-like symptoms in extremities. Clin. Rheumatol. 23(3), 246-248 (2004).

4. Sugisaki K, Hirose T. Remitting seronegative symmetrical synovitis with pitting edema (RS3PE) syndrome following spontaneous rupture of a gouty tophus. Mod. Rheumatol. 18(6), 630-633 (2008).

5. Choi YM, Sheen DH, Lee YJ et al. Sjogren's syndrome presenting as remitting seronegative symmetric synovitis with pitting edema (RS3PE). J. Korean. Med. Sci. 18(4), 606-608 (2003).

6. Matsuda M, Shimojima Y, Gono T et al. Remitting seronegative symmetrical synovitis with pitting oedema/polymyalgia rheumatica after infection with Mycoplasma pneumoniae. Ann. Rheum. Dis. 64(12), 1797-1798 (2005).

7. Salam A, Henry R, Sheeran T. Acute onset polyarthritis in older people: Is it RS3PE syndrome? Cases. J. 1(1), 132 (2008).
8. Manzo C, Natale M. Polymyalgia Rheumatica in Association with Remitting Seronegative Sinovitis with Pitting Edema: A Neoplastic Warning. Can. Geri. J. 20(2), 94-96 (2017).

9. Yao Q, Su X, Altman RD. Is remitting seronegative symmetrical synovitis with pitting edema (RS3PE) a subset of rheumatoid arthritis? Semin. Arthritis. Rheum. 40(1), 89-94 (2010).

10.McGonagle D, Pease C, Marzo-Ortega $\mathrm{H}$ et al. The case for classification of polymyalgia rheumatica and remitting seronegative symmetrical synovitis with pitting edema as primarily capsular/entheseal based pathologies. J. Rheumatol. 27(4), 837-840 (2000).

11.Cantini F, Salvarani C, Olivieri I et al. Remitting seronegative symmetrical synovitis with pitting oedema (RS3PE) syndrome: a prospective follow up and magnetic resonance imaging study. Ann. Rheum. Dis. 58(4), 230-236 (1999).

12.Arima K, Origuchi T, Tamai M et al. RS3PE syndrome presenting as vascular endothelial growth factor associated disorder. Ann. Rheum. Dis. 64(11), 1653-1655 (2005).

13.Smets P, Devauchelle-Pensec V, Rouzaire PO et al. Vascular endothelial growth factor levels and rheumatic diseases of the elderly. Arthritis. Res. Ther. 18(1), 283 (2016).

14.Sakamoto T, Ota S, Haruyama T et al. A Case of Paraneoplastic Remitting Seronegative Symmetrical Synovitis with Pitting Edema Syndrome Improved by Chemotherapy. Case. Rep. Oncol. 10(3), 1131-1137 (2017). 